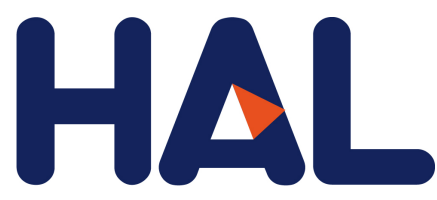

archives-ouvertes

\title{
Quantitative Imaging With Incident Field Modeling From Multistatic Measurements on Line Segments
}

\author{
Soufiane Nounouh, Christelle Eyraud, Amelie Litman, Hervé Tortel
}

\section{To cite this version:}

Soufiane Nounouh, Christelle Eyraud, Amelie Litman, Hervé Tortel. Quantitative Imaging With Incident Field Modeling From Multistatic Measurements on Line Segments. IEEE Antennas and Wireless Propagation Letters, Institute of Electrical and Electronics Engineers, 2015, 14, pp.253 256. $<10.1109 /$ LAWP.2014.2360775> $>$ hal-01279558>

\section{HAL Id: hal-01279558 \\ https://hal-amu.archives-ouvertes.fr/hal-01279558}

Submitted on 30 Oct 2018

HAL is a multi-disciplinary open access archive for the deposit and dissemination of scientific research documents, whether they are published or not. The documents may come from teaching and research institutions in France or abroad, or from public or private research centers.
L'archive ouverte pluridisciplinaire HAL, est destinée au dépôt et à la diffusion de documents scientifiques de niveau recherche, publiés ou non, émanant des établissements d'enseignement et de recherche français ou étrangers, des laboratoires publics ou privés. 


\title{
Quantitative imaging with incident field modelling from multistatic measurements on line segments
}

\author{
Soufiane Nounouh, Christelle Eyraud, Amélie Litman, and Hervé Tortel
}

\begin{abstract}
In this paper, we focus on an imaging procedure which retrieves the complex permittivity map of an investigation domain from electromagnetic scattered field measurements. The transmitter and the receiver are independently moved along two parrallel lines of finite extent. In such configuration, the antenna effects are of drastic importance. We propose thus a quantitative modeling of the antennas which does not require additional calibration process. The antennas model is completely incorporated in the inversion procedure and allows to recover tubes whose diameters are of the order of $\frac{\lambda}{5}$.
\end{abstract}

Index Terms-Microwave tomography, antenna measurement, calibration, inverse scattering, electromagnetic field scattering, antenna model, permittivity

\section{INTRODUCTION}

$\mathbf{E}$ LECTROMAGNETIC wave probing is an interesting tool to obtain the physical features of unknown targets (position, shape, size, complex permittivity). Indeed, these characteristics can be retrieved from scattered fields measurements thanks to the resolution of a non-linear inverse problem. A keypoint when dealing with quantitative imaging and with experimental data is the calibration procedure. In spherical/circular configurations, when the antennas are sufficiently far away from the target, the calibration can be performed with a single complex coefficient calculated using a reference target [1] [2]. When the antennas are moving closer to the target, even in fullaperture configurations, the impact of the antenna radiation pattern must be investigated, and more complicated calibration procedures might be required. For example, full coefficient matrices can compensate from the fact that, in antenna arrays, the illumination may differ from one antenna to the other [3]. Antennas modelling [4] [5] may also been applied, even if in [4], they conclude that a standard calibration with a reference target should be sufficient for circular configurations. In this paper, we consider a geometrical configuration where the antennas effects are of even greater importance. Indeed, both the transmitter's and the receiver's angles of sight influence the target illumination and the scattered field measurement. As in [6], we propose to properly model the radiation pattern of the antennas on the transmitting and receiving side. But instead of employing a multipolar expansion to mimic the fields, we prefer to replace each antenna by a set of elementary line sources [7] [8], as we are dealing herein with a two-dimensional configuration. A fine modelling of the directivity of the antennas is rendered possible by weighting each elementary sources according to the measured radiation

All the authors are with Aix Marseille Université, CNRS, Centrale Marseille, Institut Fresnel, UMR 7249, 13397, Marseille, France pattern of each antenna. The advantage of this antenna model is that it does not require addition calibration process: no extra network analyzer calibration, no antennas phase centers determination, no time gating, no reference target. Moreover, such a model can be easily incorporated in a finite element code [9] and enables a direct and quantitative comparisons of the measured and simulated fields. This model can be also easily incorporated in the iterative inversion algorithm, in particular in the gradients computations, in order to retrieve from the measured fields the unknown permittivity distribution of the targets. The validation of the complete procedure is performed by considering two targets composed of several tubes. Multistatic measurements are performed at a single frequency, but only the transmission part is exploited. Even if the problem is largely under-determined considering the ratio between the number of unknowns and the number of measurements, the imaging results show that it is possible to recover tubes whose diameters are of the order of $\lambda / 5$.

The paper is organized as follows. Part II is devoted to the description of the experimental study, the measurement protocol and the associated data treatments. The way the effective antennas radiation patterns are incorporated in the model of the experimental environment is presented in Section III. This forward solver, based on a home-made Finite Element solver, is then used at each step of the iterative imaging procedure which is presented in Section IV. The quantitative reconstructions obtained from the measurements are provided in Section V. Concluding remarks follow in Section VI.

\section{EXPERIMENTAL STUDY}

\section{A. Experimental setup}

This work is performed in the cubic anechoic chamber of Institut Fresnel $\left((3 \times 3 \times 3) \mathrm{m}^{3}\right)$ (Fig. 1). A multistatic scanner has been mimicked by displacing a transmitting antenna and a receiving antenna in an independent way thanks to four independent mechanical motors (position accuracy: $0.01 \mathrm{~mm}$ ). The transmitter and receiver antennas are two identical double ridged horn antennas (DRGHA, RF Consulting), linearly polarized with a $-3 \mathrm{~dB}$ aperture equal to $59^{\circ}$. During the experiment, they are kept in the same $z$-plane, facing each other, and oriented such that the electric field vector is always parallel to the $z$-axis. The transmitting antenna moves sequentially along the $y$-axis and for each of its position, an excursion along the $y$-axis is explored with the receiving antenna. The fields are measured with a Vectorial Network Analyzer (Anritsu 37369D) used in a multiple source configuration in order to improve the dynamic range. A synthesizer (Anritsu MG3694 



Fig. 1. (left) Picture and (right) Sketch of the experimental setup with transmitters and receivers positions.

A) and two external mixers (Marki Microwave M20226LP) are combined to shift the high frequency signal to an intermediate frequency just before or after the antenna. In addition, two amplifiers intensify the intermediate frequency signals. All the measurements are performed at $2.1 \mathrm{GHz}$. In this paper, the time convention is set to $\exp (-j \omega t)$.

\section{B. Measurement protocol and post-processing}

The first step consists in performing a measurement in the presence of the target - called the total field - $E_{\text {tot }}^{\text {mea }}$. Then, the object is removed and a second field without the object - called the incident field - $E_{\text {inc }}^{\text {mea }}$ is measured. No extra measurement are required. Indeed, the third step of traditional scattering measurements, which consists in measuring the field in presence of a reference target, is voluntarily skipped.

In ideal conditions, the scattered field $E_{\mathrm{sca}}^{\text {mea }}$ is obtained by a complex subtraction between the total field and the incident field. As these two fields are not measured simultaneously, deviation phenomena may occur. A weak drift error - around hundredth of radians in phase and one percent in magnitudecan create disturbances on the scattered field and thus this error has to be compensated.

We use a method based on the spectral analysis of the reduced scattered field $E_{\mathrm{sca}}^{\mathrm{mea}} e^{j \psi}$, i.e, the scattered field multiplied by a phase term which depends on the position of each receiver and on the target center [10]. The scattered field without distorsions has a limited spectral spatial bandwidth when it is observed sufficiently far from the target (above $\lambda / 2$, $\lambda$ being the free-space wavelength). For each illumination, the corrected scattered field $E_{\mathrm{sca}}^{\text {mea }} c$ is extracted as follows

$$
E_{\text {sca }}^{\text {mea }, c}=\beta e^{j \gamma} E_{\text {tot }}^{\text {mea }}-E_{\text {inc }}^{\text {mea }}
$$

The correction coefficient $\left(\beta e^{i \gamma}\right)$ is then calculated for each illumination, by minimizing the spectrum of the corrected reduced scattered field $E_{\text {sca }}^{\text {mea, } c} e^{j \psi}$ [11].

\section{FIELDS MODELLING}

As we aim at a quantitative imaging of objects in a lineconfiguration, the radiation pattern of both the transmitting and the receiving antennas have thus a substantial influence on the measured fields. Then, it is of great importance to provide an accurate modelling of the antenna radiation pattern. Moreover, in this configuration the antennas are relatively close to the object ( $3.5 \lambda$ at $2.1 \mathrm{GHz})$, it is even more important to develop an accurate model for both the illumination wave and the receiving wave. This model should satisfy the following requirements: i) versatile and independent from the experimental configuration, ii) easily adapted to various types of antennas and iii) easily implemented in our scattering model.

As the illuminated targets are cylindrical, non-magnetic and assumed to be sufficiently long along the $z$-axis, we restrain our electromagnetic model to the $(x O y)$ plane (Fig. 1).

\section{A. Incident field modelling}

One of the simplest model is to replace each antenna by a set of equivalent 2D omnidirectional line sources [7] [8]. These elementary sources are equally spaced on an equivalent aperture - larger than the real aperture of the horn antenna with a spacing less than half a wavelength for the operating frequency. Each elementary source is weighted by an associated complex coefficient. The field radiated by a transmitting antenna placed in $\mathbf{r}_{t}$ and measured by a receiving antenna placed in $\mathbf{r}_{r}$ can then be written as [9] [12]

$$
E_{\mathrm{inc}}^{\mathrm{ant}}\left(\mathbf{r}_{r} ; \mathbf{r}_{t}\right)=\left(k_{0}\left|\mathbf{r}_{t}-\mathbf{r}_{r}\right|\right)^{\alpha} \sum_{i=1}^{N_{t}} \sum_{j=1}^{N_{r}} C_{r}^{j} C_{t}^{i} E_{\mathrm{inc}}^{\mathrm{ele}}\left(\mathbf{r}_{r}^{j} ; \mathbf{r}_{t}^{i}\right)
$$

where $k_{0}$ is the free-space wavenumber. $E_{\text {inc }}^{\text {ele }}\left(\mathbf{r}_{r}^{j} ; \mathbf{r}_{t}^{i}\right)$ is the field radiated in free-space by an omnidirectional line source located at $\mathbf{r}_{t}^{i}$ and measured by an omnidirectional receiver $\mathbf{r}_{r}^{j}$. $C_{t}^{i}$ (resp. $C_{r}^{j}$ ) are the complex coefficients weighting each elementary source (resp. receiver). $N_{t}$ (resp. $N_{r}$ ) is the number of line sources necessary to model the transmitting (resp. receiving) antenna. As the two antennas are assumed to be identical, we take $N_{r}=N_{t}=N$ and $C_{r}^{i}=C_{t}^{i}$. The term $\left(k_{0}\left|\mathbf{r}_{t}-\mathbf{r}_{r}\right|\right)^{\alpha}$ corresponds to a $2 \mathrm{D} / 3 \mathrm{D}$ correcting factor, to compensate for the fact that the real antenna radiates a vectorial field while an elementary line source generates a 2D electric field. All the parameters of this model $\left(N_{r}, N_{t}\right.$, $C_{t}, C_{r}$ and $\alpha$ ) are derived by minimizing the $L_{2}$-difference $\left\|E_{\text {inc }}^{\text {meas }}-E_{\text {inc }}^{\text {ant }}\right\|$ between the measured and calculated incident fields thanks to a classical non-linear least-square optimization scheme. At the operating frequency of $2.1 \mathrm{GHz}$, the number of line sources necessary to model our antenna is found equal to $N=11$ while the $\alpha$ coefficient is found equal to -0.475 . This latter value is consistent with the $1 / \sqrt{r}$ term appearing between the $2 \mathrm{D}$ and $3 \mathrm{D}$ far-field approximation.

This modelled incident field is quantitatively comparable to the measured one, both in amplitude and in phase (Fig. 2). Instead, a single line source model (obtained by setting $N=1$ ) is not sufficient to properly imitate the measured incident field.

\section{B. Scattered field modelling}

The scattered field model follows a similar expression

$E_{\mathrm{sca}}^{\mathrm{ant}}\left(\mathbf{r}_{r} ; \mathbf{r}_{t}\right)=\left(k_{0}\left|\mathbf{r}_{t}-\mathbf{r}_{o}\right|\right)^{\alpha} \sum_{i=1}^{N_{t}} \sum_{j=1}^{N_{r}} D_{r}^{j} C_{t}^{i} E_{\mathrm{sca}}^{\mathrm{ele}}\left(\mathbf{r}_{r}^{j} ; \mathbf{r}_{t}^{i}\right)$

where $\mathbf{r}_{o}$ is the effective position of the object center and $E_{\text {sca }}^{\text {ele }}$ is the scattered field calculated with omnidirectionnal sources 

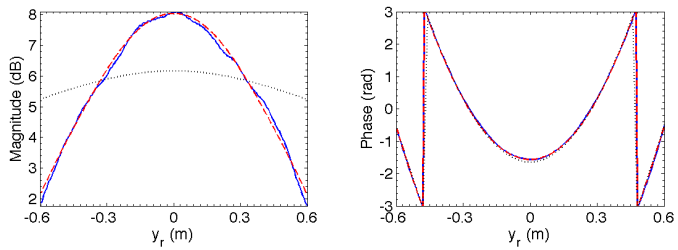

Fig. 2. Magnitude and phase of the incident field when $\mathbf{r}_{s}=(0,0,0) \mathrm{m}$ and $\mathbf{r}_{r}=\left(0.82, y_{r}, 0\right) \mathrm{m}$. (-) $E_{\mathrm{inc}}^{\mathrm{mea}},(--) E_{\text {inc }}^{\text {ant }}$ with $N=11,(\ldots) E_{\text {inc }}^{\text {ant }}$ with $N=1\left(\propto H_{0}\left(k_{0}\left|\mathbf{r}_{r}-\mathbf{r}_{t}\right|\right)\right)$.

and receivers. The difference between (Eq. 2) and (Eq. 3) relies in the receiver coefficients $D_{r}^{j}$. Indeed, these coefficients have to be modified according to the reciprocity principle as the target, being a 2D object, radiates a 2D scattered field,

$$
D_{r}^{j}=C_{t}^{j} \frac{\left(\left|\mathbf{r}_{t}-\mathbf{r}_{o}\right|+\left|\mathbf{r}_{r}-\mathbf{r}_{o}\right|\right)^{\alpha}}{\left(\left|\mathbf{r}_{r}-\mathbf{r}_{o}\right|\right)^{\alpha}}
$$

This simulated scattered field can be directly and quantitatively compared to the measured scattered field (Fig. 3) even without a classical calibration procedure based on a reference target. This field is then directly used in the imaging procedure to reach the reconstruction results presented in Section V.

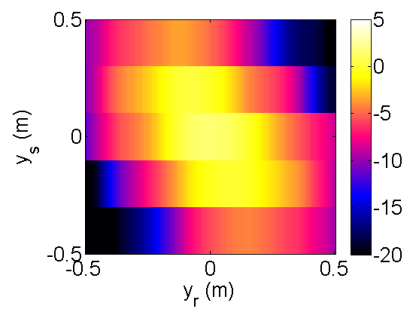

(a) $\left|E_{\text {sca }}^{\text {meas }, \mathrm{c}}\right|(\mathrm{dB})$

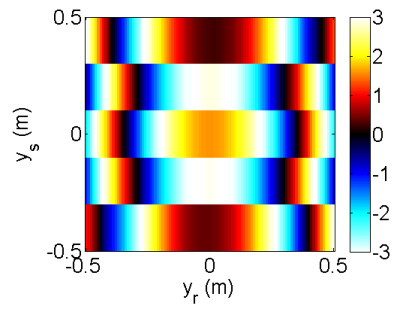

(c) $\arg \left(E_{\text {sca }}^{\text {meas,c }}\right)(\mathrm{rad})$

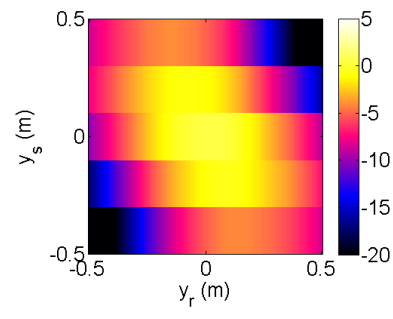

(b) $\left|E_{\text {sca }}^{\text {ant }}\right|(\mathrm{dB})$

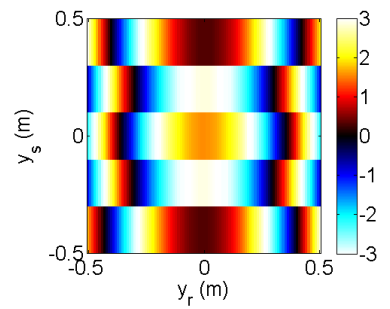

(d) $\arg \left(E_{\text {sca }}^{\text {ant }}\right)(\mathrm{rad})$
Fig. 3. Multistatic scattered field matrices for Target I (see V).

The elementary fields $E_{\text {inc }}^{\text {ele }}$ and $E_{\text {sca }}^{\text {ele }}$ are computed with a home-made Finite Element solver [13]. For this type of configuration, the typical size of the underlying linear system is around 230000 unknowns and each field is computed in few seconds for a given source position.

\section{IMAGING PROCEDURE}

The complex permittivity map of the investigated zone must now be retrieved from the measured fields. A non-linear inversion scheme has thus been implemented. To that end, a cost function $\mathcal{F}$ is introduced to appraise the discrepancy $\Delta E_{\mathrm{sca}}=E_{\mathrm{sca}}^{\mathrm{mea}, c}-E_{\mathrm{sca}}^{\text {ant }}$ between the measured scattered field $E_{\mathrm{sca}}^{\text {mea, } c}$ (corrected from the drift error) and the scattered field $E_{\mathrm{sca}}^{\mathrm{ant}}$ calculated for a given permittivity map distribution $\varepsilon_{r}(\mathbf{r})=\varepsilon_{r}^{\prime}(\mathbf{r})+j \varepsilon_{r}^{\prime \prime}(\mathbf{r})$ :

$$
\mathcal{F}=\frac{1}{2} \sum_{t}^{n_{t}} \sum_{r}^{n_{r}}\left|\Delta E_{\text {sca }}\left(\mathbf{r}_{r} ; \mathbf{r}_{t}\right)\right|^{2}
$$

where $n_{t}$ and $n_{r}$ correspond to the number of effective transmitters and receivers positions. The field $E_{\text {sca }}^{\text {ant }}$ is computed with the forward solver presented in Section III.

The cost function is iteratively minimized by changing the permittivity map with the following recurrence relation, expressed at iteration step $n$ :

$$
\varepsilon_{r}^{(n+1)}(\mathbf{r})=\varepsilon_{r}^{(n)}(\mathbf{r})+\alpha^{(n)} d^{(n)}
$$

The optimal step $\alpha^{(n)}$ is chosen to comply the Wolfe conditions [14]. The descent direction $d^{(n)}$ is derived from the Quasi-Newton Broyden-Fletcher-Goldfarb-Shanno (BFGS) method [14], which requires the cost function gradient $\nabla_{\varepsilon_{r}} \mathcal{F}$. This gradient is computed using an adjoint problem [13]

$$
\nabla_{\varepsilon_{r}} \mathcal{F}(\mathbf{r})=k_{0}^{2} \sum_{t}^{n_{t}} E_{\mathrm{tot}}^{\mathrm{sim}}\left(\mathbf{r} ; \mathbf{r}_{t}\right) P_{\mathrm{tot}}\left(\mathbf{r} ; \mathbf{r}_{t}\right)
$$

where the antenna radiation pattern is completely taken into account throughout the inverse procedure as

$P_{\text {tot }}\left(\mathbf{r} ; \mathbf{r}_{t}\right)=\sum_{r=1}^{n_{r}}\left(k_{0}\left|\mathbf{r}_{t}-\mathbf{r}_{r}\right|\right)^{\alpha} \overline{\Delta E_{\mathrm{sca}}\left(\mathbf{r}_{r} ; \mathbf{r}_{t}\right)} P_{\text {tot }}^{\text {sim }}\left(\mathbf{r} ; \mathbf{r}_{r}\right)$

with $E_{\text {tot }}^{\mathrm{sim}}\left(\mathbf{r} ; \mathbf{r}_{t}\right)=\sum_{i} C_{t}^{i} E_{\mathrm{tot}}^{\mathrm{ele}}\left(\mathbf{r} ; \mathbf{r}_{t}^{i}\right)$ and $P_{\text {tot }}^{\mathrm{sim}}\left(\mathbf{r} ; \mathbf{r}_{r}\right)=\sum_{j} D_{r}^{j} E_{\mathrm{tot}}^{\mathrm{ele}}\left(\mathbf{r} ; \mathbf{r}_{r}^{j}\right)$.

A limited-memory version of the BFGS scheme [15] is employed as there are as many unknowns as triangles present in the investigation area. Indeed, during the iterative process, only the relative permittivity values associated to a given investigation domain are updated while the other permittivity values are kept equal to 1 . Very weak information is introduced on the permittivity map, i.e., we only use positivity constraints to ensure physically realistic values $\left(\varepsilon_{r}^{\prime}(\mathbf{r}) \geq 1\right.$ and $\left.\varepsilon_{r}^{\prime \prime}(\mathbf{r}) \geq 0\right)$.

\section{Results}

Two targets are considered in this paper: one purely dielectric and one mixing dielectric and metallic parts. Target $I$ is composed of a tube made of polyurethane $\left(\varepsilon_{r}=1.45\right)$ with radius $r=0.04 \mathrm{~m}$ placed at $(0.077,0) \mathrm{m}$ and two PVC tubes $\left(\varepsilon_{r}=3.05\right)$ of radius $r=0.015 \mathrm{~m}$ placed at $(0.0283, \pm 0.0283) \mathrm{m}$. Target II is composed of two PVC tubes $\left(\varepsilon_{r}=3.05\right)$ with radius $r=0.015 \mathrm{~m}$ placed at $(0, \pm 0.09) \mathrm{m}$ and one metallic tube with radius $r=0.015 \mathrm{~m}$ placed at $(0,0) \mathrm{m}$. The scattered field is measured for $n_{t}=5$ transmitting positions and $n_{r}=101$ receiver positions, both equally spaced on a $1 \mathrm{~m}$ long line along the $y$-axis (Fig. 1). The emitting and receiving lines are respectively at $x= \pm 0.5 \mathrm{~m}$.

For the reconstructions, a circular and centred investigation domain $\Omega$ with a $0.3 \mathrm{~m}$ radius is considered and meshed with a lattice spacing equal to $0.004 \mathrm{~m}(\lambda / 35)$. This leads to 40000 complex permittivity unknowns and an inverse problem largely under-determined as the ratio between the number of unknowns and measurements is about $1 / 150$. This figure must 
be further reduced as, for the given configuration, the number of degrees of freedom is around 45 [16]. For the initial guess, $\varepsilon_{r}$ is set to 1 everywhere. The iterative process stops when the cost function $\mathcal{F}$ is below the signal-to-noise ratio deduced from the experiments [17]. The reconstructions results for

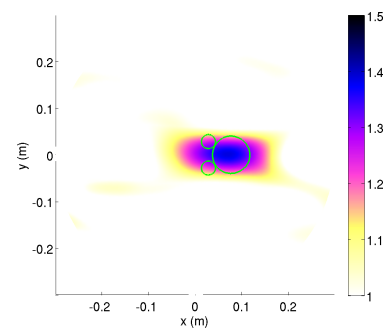

(a) Target $I-\Re\left(\varepsilon_{r}\right)$

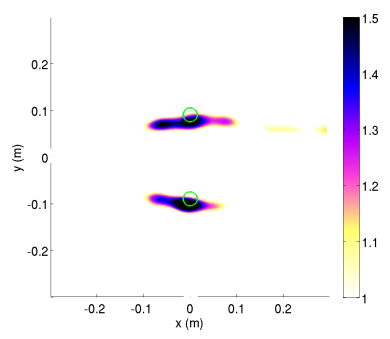

(c) Target II $-\Re\left(\varepsilon_{r}\right)$



(b) Target I - $\Im\left(\varepsilon_{r}\right)$

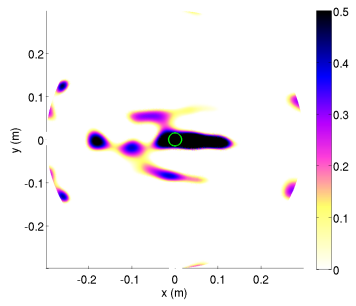

(d) Target II $-\Im\left(\varepsilon_{r}\right)$
Fig. 4. Reconstructed permittivity maps with $N=11$. The effective boundaries of the target are plotted in color.

the two targets are presented in Fig. 4. The positions of the dielectric and metallic targets are well retrieved and the targets dimensions according to the $y$-axis are well determined even if the object radius is very small w.r.t. the wavelength (the diameter of the PVC tubes corresponds to $\lambda / 5$ ). Due to the lack of measurement along the $x$-axis and to the fact that no a priori information, except for positivity, is introduced in the inversion scheme, the dimensions of the objects along the $x$-axis are overestimated (Fig. 5). This is typical from transmission measurements in an open-line configuration and this leads to an underestimation of the permittivity value - particularly for the PVC tubes. A comparison with the reconstruction results obtained using a classical calibration technique - with a single complex coefficient - is presented in Fig. 5.

As expected, taking into account the antennas radiation pattern into the modelling greatly improves the reconstructions, in terms of permittivity values and target dimensions (Fig. 5).

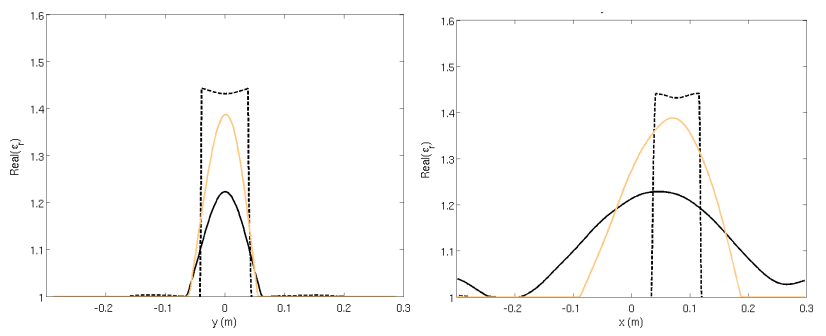

Fig. 5. Profile cuts of $\Re\left(\varepsilon_{r}\right)$ for Target $I$ with various fields models. (Left) $x=0.077 \mathrm{~m}$ (Right) $y=0 \mathrm{~m}$. (-) True profile, $(-) N=11,(-) N=1$.

\section{CONCLUSION}

In this paper, we have presented a complete quantitative imaging procedure from measurements performed on line segments. Due to the measurement configuration, the radiation pattern of the antennas must be taken into account. By representing the antennas with a set of weighted elementary sources, we are enable to directly perform quantitative comparisons between experimental and simulated fields both in terms of levels and forms. Moreover, this antenna model is completely taken into account in the quantitative inversion procedure and leads to improved reconstructed profiles. This approach does not necessitate a reference target and is simple to introduce in various numerical simulation tools, such as the finite element method. It can also be easily adapted to 3D problems.

\section{REFERENCES}

[1] J.-M. Geffrin, C. Eyraud, A. Litman, P. Sabouroux, "Optimization of a Bistatic Microwave Scattering Measurement Setup: From High to Low Scattering Targets", Radio Science, 44:RS003837, 2009.

[2] C. Eyraud, J.-M. Geffrin, A. Litman and J.-P. Spinelli, ”A large 3D target with small inner details: A difficult cocktail for imaging purposes without a-priori knowledge on the scatterers geometry", Radio Science, 47:RSOE23, 2012.

[3] A. Litman, J.-M. Geffrin and H. Tortel, "On the calibration of a multistatic scattering matrix measured by a fixed circular array of antennas", Progr. Electromagn. Res., 110:1-21, 2010.

[4] M. Ostadrahimi, P. Mojabi, C. Gilmore, A. Zakaria, S. Noghanian, S. Pistorius and J. LoVetri, "Analysis of Incident Field Modeling and Incident/Scattered Field Calibration Techniques in Microwave Tomography", IEEE Antennas Wireless Propagat. Lett., 10:900-904, 2011.

[5] M. Haynes, S. Clarkson and M. Moghaddam, "Electromagnetic Inverse Scattering Algorithm and Experiment using Absolute Source Characterization”, IEEE Trans. Antenna Propagat., 60:854-1867, 2012.

[6] L. Bellomo, S. Pioch, M. Saillard and K. Belkebir, "An improved antenna calibration methodology for microwave diffraction tomography in limitedaspect configurations", IEEE Trans. Antenna Propagat., 62:2450-2462, 2014.

[7] M. Serhir, P. Besnier and M. Drissi, "An accurate equivalent behavioural model of antenna radiation using a mode-matching technique based on spherical near field measurements", IEEE Trans. Antenna Propagat., 56:48-57, 2008.

[8] M. Serhir, J.-M. Geffrin; A. Litman, P. Besnier, "Aperture Antenna Modeling by a Finite Number of Elemental Dipoles From Spherical Field Measurements", IEEE Trans. Antennas Propagat., 58:1260-1268, 2010.

[9] S. Nounouh, C. Eyraud, H. Tortel and A. Litman, "Modeling of the antenna effects and calibration for subsurface probing", Microwave and Optical Technology Letters, vol. 56, pp. 2516 - 2522, 2014.

[10] O. Bucci, L. Crocco, M. D'Urso and T. Isernia, "Inverse scattering from phaseless measurements of the total field on open lines", J. Opt. Soc. Am. A, 23:25662577, 2006.

[11] C. Eyraud, J.-M. Geffrin, P. Sabouroux and H. Giovannini, "Drift correction for scattering measurements", Appl. Phys. Lett., 89:244104, 2006.

[12] S. Nounouh, C. Eyraud, H. Tortel and A. Litman, "Near-subsurface imaging from a multistatic/single frequency scanner", IWAGPR Proc., 1-6, 2013.

[13] R. Lencrerot, A. Litman, H.Tortel, J-M Geffrin "Measurement strategies for a confined microwave circular scanner", Inv. Probl. Science Eng., 17:787-802, 2009.

[14] J. Nocedal and S. Wright, "Numerical optimization", Springer, 2006.

[15] R. Byrd, P. Lu, J. Nocedal and C. Zhu, "A limited memory algorithm for bound constraint optimization", SIAM J. Sci. Comput., 16:1190-1208, 1995.

[16] R. Pierri and F. Soldovieri "On the information content of the radiated fields in the near zone over bounded domain", Inve. Probl., 14:321-337, 1998.

[17] S. Nounouh, "Protocoles de mesure et de calibrage de champs électromagnétiques en vue de l'imagerie par diffraction d'objets faiblement enfouis", PhD thesis, Aix-Marseille Université, 2013. 